\title{
POSITIVE SOLUTIONS AND EIGENVALUES OF CONJUGATE BOUNDARY VALUE PROBLEMS
}

\author{
by RAVI P. AGARWAL, MARTIN BOHNER* and PATRICIA J. Y. WONG
}

(Received 12th June 1997)

We consider the following boundary value problem

$$
\begin{aligned}
(-1)^{n-p} y^{(n)}+\lambda H(t, y) & =\lambda K(t, y), \quad n \geq 2, t \in(0,1) \\
y^{(i)}(0) & =0, \quad 0 \leq i \leq p-1 \\
y^{(n)}(1) & =0, \quad 0 \leq i \leq n-p-1
\end{aligned}
$$

where $\lambda>0$ and $1 \leq p \leq n-1$ is fixed. The values of $\lambda$ are characterized so that the boundary value problem has a positive solution. Further, for the case $\lambda=1$ we offer criteria for the existence of two positive solutions of the boundary value problem. Upper and lower bounds for these positive solutions are also established for special cases. Several examples are included to dwell upon the importance of the results obtained.

1991 Mathematics subject classification: 34B15.

\section{Introduction}

In this paper we shall consider the following $n$th order differential equation together with conjugate boundary conditions

$$
\text { (E) }\left\{\begin{array}{c}
(-1)^{n-p} y^{(n)}+\lambda H(t, y)=\lambda K(t, y), t \in(0,1) \\
y^{(i)}(0)=0,0 \leq i \leq p-1 ; \quad y^{(i)}(1)=0,0 \leq i \leq n-p-1
\end{array}\right.
$$

where $n \geq 2, \lambda>0$ and $p$ is a fixed integer satisfying $1 \leq p \leq n-1$. Throughout we assume that there exist continuous functions $f:[0, \infty) \rightarrow(0, \infty)$ and $k, k_{1}, h, h_{1}:(0,1) \rightarrow \mathbb{R}$ such that

(A1) $f$ is nondecreasing;

(A2) for $x \in[0, \infty)$,

$$
h(t) \leq \frac{H(t, x)}{f(x)} \leq h_{1}(t), \quad k(t) \leq \frac{K(t, x)}{f(x)} \leq k_{1}(t)
$$

- The author is grateful to Alexander von Humboldt Foundation for awarding him a Feodor Lynen Research Fellowship to support this work. 
(A3) $k(t)-h_{1}(t)$ is nonnegative and is not identically zero on any nondegenerate subinterval of $(0,1)$;

(A4) $\int_{0}^{1}[t(1-t)]^{r}\left[k_{1}(t)-h(t)\right] d t<\infty$, where $r=\min \{p, n-p\}$.

By a positive solution $y$ of $(E)$, we mean $y \in C^{(n)}(0,1)$ satisfying $(E)$, and $y$ is nonnegative and is not identically zero on [0,1]. If, for a particular $\lambda$ the boundary value problem (E) has a positive solution $y$, then $\lambda$ is called an eigenvalue and $y$ a corresponding eigenfunction of $(\mathrm{E})$. We let

$$
E=\{\lambda>0 \mid(E) \text { has a positive solution }\}
$$

be the set of eigenvalues of the boundary value problem (E). Further, we introduce the notations

$$
f_{0}=\lim _{x \rightarrow 0^{+}} \frac{f(x)}{x}, \quad f_{\infty}=\lim _{x \rightarrow \infty} \frac{f(x)}{x}
$$

First, we shall characterize the values of $\lambda$ for which the boundary value problem (E) has a positive solution. To be specific, we shall show that the set $E$ is an interval and establish conditions under which $E$ is a bounded or an unbounded interval. Further, on relaxing the monotonicity condition (A1), explicit eigenvalue intervals are obtained in terms of $f_{0}$ and $f_{\infty}$.

Next, for $\lambda=1$ we shall investigate the existence of two positive solutions of (E). In addition to the existence criteria developed, we shall consider the following special cases of $(\mathrm{E})(n=2, p=1)$

$$
\left(\mathrm{E}_{1}\right) \quad y^{\prime \prime}+a(t)\left(y^{\alpha}+y^{\beta}\right)=0, t \in(0,1) ; \quad y(0)=y(1)=0
$$

and

$$
\left(\mathrm{E}_{2}\right) \quad y^{\prime \prime}+a(t) e^{a y}=0, t \in(0,1) ; \quad y(0)=y(1)=0 .
$$

It is assumed that $0 \leq \alpha<1<\beta, \sigma>0$, and $a(t) \in C[0,1]$ is nonnegative and is not identically zero on any nondegenerate subinterval of $(0,1)$. Other than providing conditions under which $\left(\mathrm{E}_{1}\right)$ and $\left(\mathrm{E}_{2}\right)$ have double positive solutions, we also establish upper and lower bounds for these positive solutions. It is noted that the importance of $\left(E_{1}\right)$ and of the discrete version of its particular cases have been well illustrated in [22] and [5] respectively. With $a(t)$ being a constant function, the boundary value problem $\left(\mathrm{E}_{2}\right)$ actually arises in applications involving the diffusion of heat generated by positive temperaturedependent sources [1]. For instance, if $\sigma=1$ the boundary value problem occurs in the analysis of Joule losses in electrically conducting solids as well as in frictional heating.

The motivation for the present work stems from many recent investigations. In fact, when $n=2$ the boundary value problem (E) models a wide spectrum of nonlinear phenomena such as gas diffusion through porous media, nonlinear diffusion generated 
by nonlinear sources, thermal selfignition of a chemically active mixture of gases in a vessel, catalysis theory, chemically reacting systems, adiabatic tubular reactor processes, as well as concentration in chemical or biological problems, where only positive solutions are meaningful, e.g., see $[4,8,10,11,18,21,32]$. For the special case $\lambda=1$, (E) and its particular and related cases have been the subject matter of many recent publications on singular boundary value problems, for this we refer to $[2,3,9$, $20,25,26,31,37]$. Further, in the case of second order boundary value problems, (E) occurs in applications involving nonlinear elliptic problems in annular regions, e.g., see $[6,7,19,34]$. Once again in all these applications, it is frequent that only solutions that are positive are useful.

Recently, several eigenvalue characterizations for particular cases of (E) have been carried out. To cite a few examples, Fink, Gatica and Hernandez [17] have dealt with the boundary value problem

$$
y^{\prime \prime}+\lambda q(t) f(y)=0, t \in(0,1) ; \quad y(0)=y(1)=0 .
$$

Another problem, namely,

$$
y^{(n)}+q(t) f(y)=0, t \in(0,1) ; \quad y^{(i)}(0)=y(1)=0,0 \leq i \leq n-2
$$

has been tackled in [12]. Further, Eloe and Henderson [13] have established some eigenvalue intervals for a special case of (E) which are improved in the present paper. As for twin positive solutions, several studies on boundary value problems different from (E) can be found in $[5,14,28,29,30]$. Our results not only generalize and extend the known theorems for all the above eigenvalue problems, but also complement the work of many authors $[3,15,16,24,33,35,36,38,39,40,41,42]$, as well as include several other known criteria offered in [1].

The outline of the paper is as follows. In Section 2 we shall state a fixed point theorem due to Krasnosel'skii [27], and present some properties of certain Green's function which are needed later. By defining an appropriate Banach space and cone, in Section 3 we shall characterize the set $E$. Explicit eigenvalue intervals in terms of $f_{0}$ and $f_{\infty}$ are established in Section 4. The investigation of the existence of double positive solutions is carried out in Section 5. Finally, the boundary value problems $\left(E_{1}\right)$ and $\left(E_{2}\right)$ are treated, respectively, in Sections 6 and 7.

\section{Preliminaries}

Theorem 2.1 ([27]). Let $B$ be a Banach space, and let $C(C B)$ be a cone. Assume $\Omega_{1}, \Omega_{2}$ are open subsets of $B$ with $0 \in \Omega_{1}, \bar{\Omega}_{1} \subset \Omega_{2}$, and let

$$
S: C \cap\left(\bar{\Omega}_{2} \backslash \Omega_{1}\right) \rightarrow C
$$

be a completely continuous operator such that, either 


\section{RAVI P. AGARWAL, MARTIN BOHNER AND PATRICIA J. Y. WONG}

(a) $\|S y\| \leq\|y\|, y \in C \cap \partial \Omega_{1}$, and $\|S y\| \geq\|y\|, y \in C \cap \partial \Omega_{2}$, or

(b) $\|S y\| \geq\|y\|, y \in C \cap \partial \Omega_{1}$, and $\|S y\| \leq\|y\|, y \in C \cap \partial \Omega_{2}$.

Then, $S$ has a fixed point in $C \cap\left(\bar{\Omega}_{2} \backslash \Omega_{1}\right)$.

To obtain a solution for (E), we require a mapping whose kernel $G(t, s)$ is the Green's function of the boundary value problem

$$
y^{(n)}=0 ; \quad y^{(i)}(0)=0,0 \leq i \leq p-1 ; \quad y^{(i)}(1)=0,0 \leq i \leq n-p-1
$$

where $1 \leq p \leq n-1$ is fixed. The Green's function $G(t, s)$ can be explicitly expressed as [23]

$$
G(t, s)=\left\{\begin{array}{l}
\sum_{j=0}^{p-1}\left[\sum_{i=0}^{p-1-j}\left(\begin{array}{c}
n-p+i-1 \\
i
\end{array}\right) t^{i}\right] \frac{t^{j}(-s)^{n-j-1}}{j !(n-j-1) !}(1-t)^{n-p}, 0 \leq s \leq t \leq 1 \\
-\sum_{j=0}^{n-p-1}\left[\sum_{i=0}^{n-p-1-j}\left(\begin{array}{c}
p+i-1 \\
i
\end{array}\right)(1-t)^{i}\right] \frac{(t-1)^{j}(1-s)^{n-j-1}}{j !(n-j-1) !} t^{p}, 0 \leq t \leq s \leq 1 .
\end{array}\right.
$$

Further, it is known that [1]

$$
(-1)^{n-p} G(t, s)>0,(t, s) \in(0,1) \times(0,1) .
$$

For each $s \in[0,1]$, we shall denote

$$
\|G(\cdot, s)\|=\sup _{t \in[0,1]}|G(t, s)|=\sup _{t \in[0,1]}(-1)^{n-p} G(t, s) .
$$

Lemma 2.1 [43]. For any $\delta \in(0,1 / 2)$ and $t \in[\delta, 1-\delta]$, we have

$$
(-1)^{n-p} G(t, s) \geq \theta\|G(\cdot, s)\|
$$

where $0<\theta<1$ is a constant given by

$$
\theta=\min \{b(p) \cdot \min \{c(p), c(n-p-1)\}, b(p-1) \cdot \min \{c(p-1), c(n-p)\}\},
$$

and the functions $b$ and $c$ are defined as

$$
b(x)=\frac{(n-1)^{n-1}}{x^{x}(n-x-1)^{n-x-1}} \text { and } c(x)=\delta^{x}(1-\delta)^{n-x-1} .
$$


Lemma 2.2. Let $q=\max \{p, n-p\}$ and $r=\min \{p, n-p\}$. For $(t, s) \in[0,1] \times[0,1]$, we have

$$
(-1)^{n-p} G(t, s) \leq \frac{2^{q-1}}{(q-1) !(n-q) !}[s(1-s)]^{r} \equiv \phi(s)
$$

Proof. For $(t, s) \in[0,1] \times[0,1]$, it is clear from (2.1) and (2.2) that

$$
\begin{aligned}
& (-1)^{n-p} G(t, s)=|G(t, s)| \\
& \leq \begin{cases}\sum_{j=0}^{p-1}\left[\sum_{i=0}^{p-1-j}\left(\begin{array}{c}
n-p+i-1 \\
i
\end{array}\right)\right] \frac{s^{n-(p-1)-1}}{j !(n-j-1) !}(1-s)^{n-p}, & s \leq t \\
\sum_{j=0}^{n-p-1}\left[\sum_{i=0}^{n-p-1-j}\left(\begin{array}{c}
p+i-1 \\
i
\end{array}\right)\right] \frac{(1-s)^{n-(n-p-1)-1}}{j !(n-j-1) !} s^{p}, & t \leq s\end{cases} \\
& \leq[s(1-s)]^{r} \cdot \max \left\{\sum_{j=0}^{p-1}\left[\sum_{i=0}^{p-1-j}\left(\begin{array}{c}
n-p+i-1 \\
i
\end{array}\right)\right] \frac{1}{j !(n-j-1) !},\right. \\
& \left.\sum_{j=0}^{n-p-1}\left[\sum_{i=0}^{n-p-1-j}\left(\begin{array}{c}
p+i-1 \\
i
\end{array}\right)\right] \frac{1}{j !(n-j-1) !}\right\} \\
& =[s(1-s)]^{r} \cdot \max \left\{\sum_{j=0}^{p-1}\left(\begin{array}{l}
n-j-1 \\
p-j-1
\end{array}\right) \frac{1}{j !(n-j-1) !},\right. \\
& \left.\sum_{j=0}^{n-p-1}\left(\begin{array}{c}
n-j-1 \\
n-p-j-1
\end{array}\right) \frac{1}{j !(n-j-1) !}\right\} \\
& =[s(1-s)]^{r} \cdot \max \left\{\frac{2^{p-1}}{(p-1) !(n-p) !}, \frac{2^{n-p-1}}{p !(n-p-1) !}\right\}=\phi(s) .
\end{aligned}
$$

We shall need the following notations later: Let

$$
v(t)=k_{1}(t)-h(t) \text { and } u(t)=k(t)-h_{1}(t)
$$

For a nonnegative $y$ on $[0,1]$, we denote

$$
m_{1}=\int_{0}^{1} \phi(s) v(s) f(y(s)) d s \text { and } m_{2}=\int_{0}^{1}\|G(\cdot, s)\| u(s) f(y(s)) d s .
$$


In view of (2.3), Lemma 2.2, (A2) and (A3), it is clear that $m_{1} \geq m_{2}>0$. Further, we define the constant $\gamma \in(0,1)$ by

$$
\gamma=\frac{\theta m_{2}}{m_{1}}
$$

\section{Eigenvalue characterization}

Let the Banach space

$$
B=\{y \mid y \in C[0,1]\}
$$

be equipped with norm $\|y\|=\sup _{\imath \in[0,1]}|y(t)|$. For a given $\delta \in\left(0, \frac{1}{2}\right)$, let

$$
C_{\delta}=\left\{y \in B \mid y(t) \text { is nonnegative on }[0,1] ; \min _{t \in\{\delta, 1-\delta]} y(t) \geq \gamma\|y\|\right\} .
$$

We note that $C_{\delta}$ is a cone in $B$. Further, let

$$
C_{\delta}(M)=\left\{y \in C_{\delta} \mid\|y\| \leq M\right\} .
$$

We define the operator $S: C_{\delta} \rightarrow B$ by

$$
S y(t)=\int_{0}^{1}(-1)^{n-p} G(t, s)[K(s, y)-H(s, y)] d s, t \in[0,1] .
$$

To obtain a positive solution of (E), we shall seek a fixed point of the operator $\lambda S$ in the cone $C_{\delta}$. It is clear from (A2) that

$$
U y(t) \leq S y(t) \leq V y(t), t \in[0,1],
$$

where

$$
U y(t)=\int_{0}^{1}(-1)^{n-p} G(t, s) u(s) f(y(s)) d s
$$

and

$$
V y(t)=\int_{0}^{1}(-1)^{n-p} G(t, s) v(s) f(y(s)) d s .
$$

We shall now show that the operator $S$ is compact on the cone $C_{\delta}$. Let us consider the case when $u(t)$ is unbounded in a deleted right neighbourhood of 0 and also in a 
deleted left neighbourhood of 1 . Clearly, $v(t)$ is also unbounded near 0 and 1 . For $m \in\{1,2,3, \cdots\}$, define $u_{m}, v_{m}:[0,1] \rightarrow \mathbb{R}$ by

$$
u_{m}(t)=\left\{\begin{array}{ll}
u\left(\frac{1}{m+1}\right), & 0 \leq t \leq \frac{1}{m+1} \\
u(t), & \frac{1}{m+1} \leq t \leq \frac{m}{m+1} \\
u\left(\frac{m}{m+1}\right), & \frac{m}{m+1} \leq t \leq 1,
\end{array} \quad v_{m}(t)= \begin{cases}v\left(\frac{1}{m+1}\right), & 0 \leq t \leq \frac{1}{m+1} \\
v(t), & \frac{1}{m+1} \leq t \leq \frac{m}{m+1} \\
v\left(\frac{m}{m+1}\right), & \frac{m}{m+1} \leq t \leq 1,\end{cases}\right.
$$

and the operators $U_{m}, V_{m}: C_{\delta} \rightarrow B$ by

$$
\begin{aligned}
& U_{m} y(t)=\int_{0}^{1}(-1)^{n-p} G(t, s) u_{m}(s) f(y(s)) d s, \\
& V_{m} y(t)=\int_{0}^{1}(-1)^{n-p} G(t, s) v_{m}(s) f(y(s)) d s .
\end{aligned}
$$

It is standard that for each $m$, both $U_{m}$ and $V_{m}$ are compact operators on $C_{\delta}$. Let $M>0$ and $y \in C_{\delta}(M)$. Then, in view of (Al) and Lemma 2.2, we find

$$
\begin{aligned}
\left|V_{m} y(t)-V y(t)\right| & \leq \int_{0}^{1}(-1)^{n-p} G(t, s)\left|v_{m}(s)-v(s)\right| f(y(s)) d s \\
& \leq f(M)\left[\int_{0}^{\frac{1}{m+1}} \phi(s)\left|v\left(\frac{1}{m+1}\right)-v(s)\right| d s+\int_{\frac{m}{m+1}}^{1} \phi(s)\left|v\left(\frac{m}{m+1}\right)-v(s)\right| d s\right] .
\end{aligned}
$$

The integrability of $\phi(t) v(t)$ (condition (A4)) implies that $V_{m}$ converges uniformly to $V$ on $C_{\delta}(M)$. Hence, $V$ is compact on $C_{\delta}$. Similarly, we can verify that $U_{m}$ converges uniformly to $U$ on $C_{\delta}(M)$ and therefore $U$ is compact on $C_{\delta}$. It follows from inequality (3.1) that the operator $S$ is compact on $C_{\delta}$.

Theorem 3.1. There exists $c>0$ such that the interval $(0, c] \subseteq E$.

Proof. Let $M>0$ be given. Define

$$
c=\frac{M}{f(M)}\left[\int_{0}^{1} \phi(s) v(s) d s\right]^{-1} .
$$

Let $\lambda \in(0, c]$. We shall prove that $(\lambda S)\left(C_{\delta}(M)\right) \subseteq C_{\delta}(M)$. For this, let $y \in C_{\delta}(M)$ and we shall first show that $\lambda S y \in C_{\delta}$. From (3.1) and (A3),

$$
(\lambda S y)(t) \geq \lambda \int_{0}^{1}(-1)^{n-p} G(t, s) u(s) f(y(s)) d s \geq 0, t \in[0,1] .
$$

Further, it follows from (3.1) and Lemma 2.2 that for $t \in[0,1]$ 


$$
S y(t) \leq \int_{0}^{1}(-1)^{n-p} G(t, s) v(s) f(y(s)) d s \leq \int_{0}^{1} \phi(s) v(s) f(y(s)) d s=m_{1} .
$$

Thus,

$$
\|S y\| \leq m_{1} .
$$

Now, on using (3.1), Lemma 2.1, (3.4) and (2.4), we find for $t \in[\delta, 1-\delta]$,

$$
\begin{aligned}
(\lambda S y)(t) & \geq \lambda \int_{0}^{1} \theta\|G(\cdot, s)\| u(s) f(y(s)) d s=\lambda \theta m_{2} \\
& \geq \lambda \theta m_{2} \frac{\|S y\|}{m_{1}}=\lambda \gamma\|S y\|=\gamma\|\lambda S y\| .
\end{aligned}
$$

Therefore,

$$
\min _{t \in[\delta, 1-\delta]}(\lambda S y)(t) \geq \gamma\|\lambda S y\| .
$$

Inequalities (3.3) and (3.5) lead to $\lambda S y \in C_{\delta}$.

Next, we shall show that $\|\lambda S y\| \leq M$. For this, on using (3.1), Lemma 2.2, (A1) and (3.2) successively, we get for $t \in[0,1]$

$$
(\lambda S y)(t) \leq c \int_{0}^{1} \phi(s) v(s) f(M) d s=M,
$$

which implies $\|\lambda S y\| \leq M$. Hence, $(\lambda S)\left(C_{\delta}(M)\right) \subseteq C_{\delta}(M)$. Also, the standard arguments yield that $\lambda S$ is completely continuous. By Schauder fixed point theorem, $\lambda S$ has a fixed point in $C_{\delta}(M)$. Clearly, this fixed point is a positive solution of $(E)$ and therefore $\lambda$ is an eigenvalue of (E). Since $\lambda \in(0, c]$ is arbitrary, it follows immediately that the interval $(0, c] \subseteq E$.

The next theorem makes use of the monotonicity and compactness of the operator $S$ on the cone $C_{\delta}$. We refer to [17, Theorem 3.2] for its proof.

Theorem 3.2 [17]. If $\lambda_{0} \in E$, then $\left(0, \lambda_{0}\right] \subseteq E$. So $E$ is an interval.

We shall establish conditions under which $E$ is a bounded or unbounded interval. For this, we need the following results.

Theorem 3.3. Let $\lambda$ be an eigenvalue of (E) and $y \in C_{\delta}$ be a corresponding eigenfunction. Further, let $d=\|y\|$. Then, 


$$
\lambda \geq \frac{d}{f(d)}\left[\int_{0}^{1} \phi(s) v(s) d s\right]^{-1}
$$

and

$$
\lambda \leq \frac{d}{f(\gamma d)}\left[\int_{\delta}^{1-\delta}(-1)^{n-p} G\left(\frac{1}{2}, s\right) u(s) d s\right]^{-1}
$$

Proof. First, for proving (3.6), we let $t_{0} \in[0,1]$ be such that $d=\|y\|=y\left(t_{0}\right)$. Then, applying (3.1), Lemma 2.2 and (A1) we find

$$
\begin{aligned}
d & =y\left(t_{0}\right)=(\lambda S y)\left(t_{0}\right) \leq \lambda \int_{0}^{1}(-1)^{n-p} G\left(t_{0}, s\right) v(s) f(y(s)) d s \\
& \leq \lambda \int_{0}^{1} \phi(s) v(s) f(y(s)) d s \leq \lambda f(d) \int_{0}^{1} \phi(s) v(s) d s
\end{aligned}
$$

from which (3.6) follows. Next, using (3.1) and $\min _{t \in[\delta . l-\delta]} y(t) \geq \gamma d$, we get

$$
\begin{aligned}
d & \geq y\left(\frac{1}{2}\right) \geq \lambda \int_{0}^{1}(-1)^{n-p} G\left(\frac{1}{2}, s\right) u(s) f(y(s)) d s \\
& \geq \lambda f(\gamma d) \int_{\delta}^{1-\delta}(-1)^{n-p} G\left(\frac{1}{2}, s\right) u(s) d s
\end{aligned}
$$

which gives (3.7) readily.

Theorem 3.4. Let

$$
\begin{gathered}
F_{B}=\left\{f \mid \frac{x}{f(x)} \text { is bounded for } x \in[0, \infty)\right\} \\
F_{0}=\left\{f \mid \lim _{x \rightarrow \infty} \frac{x}{f(x)}=0\right\} \quad \text { and } \quad F_{\infty}=\left\{f \mid \lim _{x \rightarrow \infty} \frac{x}{f(x)}=\infty\right\} .
\end{gathered}
$$

(a) If $f \in F_{B}$, then $E=(0, c)$ or $(0, c]$ for some $c \in(0, \infty)$.

(b) If $f \in F_{0}$, then $E=(0, c]$ for some $c \in(0, \infty)$.

(c) If $f \in F_{\infty}$, then $E=(0, \infty)$.

Proof. First, (a) is immediate from (3.7). Next, we prove (b). Since $F_{0} \subseteq F_{B}$, it follows from Case (a) that $E=(0, c)$ or $(0, c]$ for some $c \in(0, \infty)$. In particular, $c=\sup E$. Let $\left\{\lambda_{m}\right\}_{m=1}^{\infty}$ be a monotonically increasing sequence in $E$ which converges 
to $c$, and let $\left\{y_{m}\right\}_{m=1}^{\infty}$ in $C_{\delta}$ be a corresponding sequence of eigenfunctions. Further, let $d_{m}=\left\|y_{m}\right\|$. Then, (3.7) implies that no subsequence of $\left\{d_{m}\right\}_{m=1}^{\infty}$ can diverge to infinity. Thus, there exists $M>0$ such that $d_{m} \leq M$ for all $m$. So $y_{m}$ is uniformly bounded. Hence, there is a subsequence of $\left\{y_{m}\right\}_{m=1}^{\infty}$, relabelled as the original sequence, which converges uniformly to some $y \in C_{\delta}$. Noting that $\lambda_{m} S y_{m}=y_{m}$, we have

$$
c S y_{m}=\frac{c}{\lambda_{m}} y_{m}
$$

Since $\left\{c S y_{m}\right\}_{m=1}^{\infty}$ is relatively compact, $y_{m}$ converges to $y$ and $\lambda_{m}$ converges to $c$, letting $m \rightarrow \infty$ in (3.8) gives $c S y=y$, i.e., $c \in E$. This completes the proof for Case (b). Finally, (c) follows from Theorem 3.2 and (3.6).

Example 3.1. Let $\lambda>0, a \geq 0$, and consider the boundary value problem

$$
y^{(4)}=\lambda \frac{(y+8)^{a}}{\left[t^{2}(1-t)^{2}+8\right]^{a}}, t \in(0,1) ; \quad y(0)=y^{\prime}(0)=y(1)=y^{\prime}(1)=0 .
$$

Here, $n=4, p=2$, and we let $f(y)=(y+8)^{a}, K(t, y)=\frac{f(y)}{\left.r^{2}(1-t)^{2}+8\right)^{a}}, H(t, y)=0$. Hence, we may take $k(t)=k_{1}(t)=\frac{K(t, y)}{f(y)}$ and $h(t)=h_{1}(t)=0$. All the hypotheses (A1)-(A4) are satisfied.

Case 1: $0 \leq a<1$. Since $f \in F_{\infty}$, by Theorem 3.4(c) the set $E=(0, \infty)$. For instance, when $\lambda=24$, the boundary value problem has a positive solution given by $y(t)=t^{2}(1-t)^{2}$.

Case 2: $a=1$. Since $f \in F_{B}$, by Theorem 3.4(a) the set $E$ is an open or a half-closed interval. Further, from Case 1 and Theorem 3.2 we note that $E$ contains the interval $(0,24]$.

Case 3: $a>1$. Since $f \in F_{0}$, by Theorem 3.4(b) the set $E$ is a half-closed interval. Again, as in Case 2 it is noted that $(0,24] \subseteq E$.

\section{Eigenvalue intervals}

For the rest of the paper, we shall not require conditions (A1) and (A4). However, we need the functions $k, k_{1}, h$ and $h_{1}$ to be continuous on the closed interval $[0,1]$.

The number $t^{*} \in[0,1]$ is defined by

$$
\int_{\delta}^{1-\delta}(-1)^{n-p} G\left(t^{*}, s\right) u(s) d s=\sup _{t \in[0,1]} \int_{\delta}^{1-\delta}(-1)^{n-p} G(t, s) u(s) d s .
$$

Theorem 4.1. Suppose that (A2) and (A3) hold. Let $\delta \in(0,1 / 2)$. Then, 


$$
\left(\frac{1}{f_{\infty} \theta \int_{\delta}^{1-\delta}(-1)^{n-p} G\left(t^{*}, s\right) u(s) d s}, \frac{1}{f_{0} \int_{0}^{1} \phi(s) v(s) d s}\right) \subseteq E .
$$

Proof. Assume $\left\{f_{\infty} \theta \int_{\delta}^{1-\delta}(-1)^{n-p} G\left(t^{*}, s\right) u(s) d s\right\}^{-1}<\lambda<\left\{f_{0} \int_{0}^{1} \phi(s) v(s) d s\right\}^{-1}$. Noting that $\gamma \leq \theta$, we let $\epsilon>0$ be such that

$$
\frac{1}{\left(f_{\infty}-\epsilon\right) \gamma \int_{\delta}^{1-\delta}(-1)^{n-p} G\left(t^{*}, s\right) u(s) d s} \leq \lambda \leq \frac{1}{\left(f_{0}+\epsilon\right) \int_{0}^{1} \phi(s) v(s) d s} .
$$

Next, we choose $w>0$ so that

$$
f(x) \leq\left(f_{0}+\epsilon\right) x, 0<x \leq w .
$$

Let $y \in C_{\delta}$ be such that $\|y\|=w$. Then, applying (3.1), Lemma 2.2, (4.2) and (4.1) successively, we find for $t \in[0,1]$,

$$
(\lambda S y)(t) \leq \lambda \int_{0}^{1} \phi(s) v(s) f(y(s)) d s \leq \lambda \int_{0}^{1} \phi(s) v(s)\left(f_{0}+\epsilon\right) y(s) d s \leq\|y\| .
$$

Hence,

$$
\|\lambda S y\| \leq\|y\| .
$$

If we set $\Omega_{1}=\{y \in B \mid\|y\|<w\}$, then (4.3) holds for $y \in C_{\delta} \cap \partial \Omega_{1}$.

Further, let $T>0$ be such that

$$
f(x) \geq\left(f_{\infty}-\epsilon\right) x, x \geq T .
$$

Let $y \in C_{\delta}$ be such that $\|y\|=T^{\prime} \equiv \max \left\{2 w, \frac{T}{y}\right\}$. Then, for $t \in[\delta, 1-\delta]$, we have $y(t) \geq \gamma\|y\| \geq \gamma \cdot \frac{T}{\gamma}=T$, which in view of (4.4) leads to

$$
f(y(t)) \geq\left(f_{\infty}-\epsilon\right) y(t), t \in[\delta, 1-\delta] .
$$

Using (3.1), (4.5) and (4.1), we find

$$
\begin{aligned}
(\lambda S y)\left(t^{*}\right) & \geq \lambda \int_{\delta}^{1-\delta}(-1)^{n-p} G\left(t^{*}, s\right) u(s) f(y(s)) d s \\
& \geq \lambda \int_{\delta}^{1-\delta}(-1)^{n-p} G\left(t^{*}, s\right) u(s)\left(f_{\infty}-\epsilon\right) y(s) d s \\
& \geq \lambda \int_{\delta}^{1-\delta}(-1)^{n-p} G\left(t^{*}, s\right) u(s)\left(f_{\infty}-\epsilon\right) \gamma\|y\| d s \geq\|y\| .
\end{aligned}
$$


Therefore,

$$
\|\lambda S y\| \geq\|y\| .
$$

If we set $\Omega_{2}=\left\{y \in B \mid\|y\|<T^{\prime}\right\}$, then (4.6) holds for $y \in C_{\delta} \cap \partial \Omega_{2}$.

Now that we have obtained (4.3) and (4.6), it follows from Theorem 2.1 that $\lambda S$ has a fixed point $y \in C_{\delta} \cap\left(\bar{\Omega}_{2} \backslash \Omega_{1}\right)$ such that $w \leq\|y\| \leq T^{\prime}$. Obviously, this $y$ is a positive solution of $(E)$.

Theorem 4.2. Suppose that (A2) and (A3) hold. Let $\delta \in(0,1 / 2)$. Then,

$$
\left(\frac{1}{f_{0} \theta \int_{\delta}^{1-\delta}(-1)^{n-p} G\left(t^{*}, s\right) u(s) d s}, \frac{1}{f_{\infty} \int_{0}^{1} \phi(s) v(s) d s}\right) \subseteq E .
$$

Proof. Assume $\left\{f_{0} \theta \int_{\delta}^{1-\delta}(-1)^{n-p} G\left(t^{*}, s\right) u(s) d s\right\}^{-1}<\lambda<\left\{f_{\infty} \int_{0}^{1} \phi(s) v(s) d s\right\}^{-1}$. Again, in view of the inequality $\gamma \leq \theta$, let $\epsilon>0$ be such that

$$
\frac{1}{\left(f_{0}-\epsilon\right) \gamma \int_{\delta}^{1-\delta}(-1)^{n-p} G\left(t^{*}, s\right) u(s) d s} \leq \lambda \leq \frac{1}{\left(f_{\infty}+\epsilon\right) \int_{0}^{1} \phi(s) v(s) d s} .
$$

Let $\bar{w}>0$ be such that

$$
f(x) \geq\left(f_{0}-\epsilon\right) x, 0<x \leq \bar{w} .
$$

Further, let $y \in C_{\delta}$ be such that $\|y\|=\bar{w}$. Then, on using (3.1), (4.8) and (4.7) successively, we get

$$
(\lambda S y)\left(t^{*}\right) \geq \lambda \int_{\delta}^{1-\delta}(-1)^{n-p} G\left(t^{*}, s\right) u(s)\left(f_{0}-\epsilon\right) y(s) d s \geq\|y\|
$$

so that (4.6) follows. If we set $\Omega_{1}=\{y \in B \mid\|y\|<\bar{w}\}$, then (4.6) holds for $y \in C_{\delta} \cap \partial \Omega_{1}$.

Next, we may choose $\bar{T}>0$ such that

$$
f(x) \leq\left(f_{\infty}+\epsilon\right) x, x \geq \bar{T} .
$$

There are two cases to consider, namely, $f$ is bounded and $f$ is unbounded.

First, suppose that $f$ is bounded, i.e., there exists some $M>0$ such that

$$
f(x) \leq M, x \in[0, \infty)
$$

We define 


$$
T_{1}=\max \left\{2 \bar{w}, \lambda M \int_{0}^{1} \phi(s) v(s) d s\right\}
$$

Let $y \in C_{\delta}$ be such that $\|y\|=T_{1}$. For $t \in[0,1]$, from (3.1), Lemma 2.2 and (4.10) we find

$$
(\lambda S y)(t) \leq \lambda \int_{0}^{1} \phi(s) v(s) f(y(s)) d s \leq T_{1}=\|y\|
$$

Hence, (4.3) holds.

Next, suppose that $f$ is unbounded. Then, there exists $T_{1}>\max \{2 \bar{w}, \bar{T}\}$ such that

$$
f(x) \leq f\left(T_{1}\right), 0<x \leq T_{1}
$$

Let $y \in C_{\delta}$ be such that $\|y\|=T_{1}$. Then, applying (3.1), Lemma 2.2, (4.11), (4.9) and (4.7) successively gives for $t \in[0,1]$

$$
(\lambda S y)(t) \leq \lambda \int_{0}^{1} \phi(s) v(s) f\left(T_{1}\right) d s \leq \lambda \int_{0}^{1} \phi(s) v(s)\left(f_{\infty}+\epsilon\right)\|y\| d s \leq\|y\|,
$$

from which (4.3) follows immediately.

In both cases, if we set $\Omega_{2}=\left\{y \in B \mid\|y\|<T_{1}\right\}$, then (4.3) holds for $y \in C_{\delta} \cap \partial \Omega_{2}$.

Now that we have obtained (4.6) and (4.3), it follows from Theorem 2.1 that $\lambda S$ has a fixed point $y \in C_{\delta} \cap\left(\bar{\Omega}_{2} \backslash \Omega_{1}\right)$ such that $\bar{w} \leq\|y\| \leq T_{1}$. It is clear that this $y$ is a positive solution of $(\mathrm{E})$.

Remark 4.1. If $f$ is superlinear (i.e., $f_{0}=0$ and $f_{\infty}=\infty$ ) or sublinear (i.e., $f_{0}=\infty$ and $\left.f_{\infty}=0\right)$, then we conclude from Theorems 4.1 and 4.2 that $E=(0, \infty)$, i.e., the boundary value problem (E) has a positive solution for any $\lambda>0$.

Also, note that Theorems 4.1 and 4.2 have improved the results in [13]. The improvement is due to the best possible bound obtained in Lemma 2.1 .

Example 4.1. Let $\lambda>0, a \leq 1$, and consider the boundary value problem

$$
-y^{(3)}=\lambda \frac{1}{\left[3 t^{2}(1-t)+6\right]^{a}}(3 y+6)^{a}, t \in(0,1) ; \quad y(0)=y^{\prime}(0)=y(1)=0 .
$$

Here, $n=3, \quad p=2$. Choosing $f(y)=(3 y+6)^{a}$, we may take $k(t)=k_{1}(t)=$ $\left[3 t^{2}(1-t)+6\right]^{-a}$ and $h(t)=h_{1}(t)=0$. The hypotheses (A2) and (A3) are satisfied.

Case I: $a<1$. It is clear that $f$ is sublinear. Hence, in view of Remark 4.1, for any $\lambda>0$ the boundary value problem has a positive solution. In fact, we note that when $\lambda=6$, one such solution is given by $y(t)=t^{2}(1-t)$.

Case 2: $a=1$. Here, $f_{0}=\infty$ and $f_{\infty}=3$. Further, we find $\phi(s)=2 s(1-s)$ and subsequently $\int_{0}^{1} \phi(s) v(s) d s=0.0529$. Hence, it follows from Theorem 4.2 that 
$(0,6.30) \subseteq E$. As an example, when $\lambda=6 \in(0,6.30)$, the corresponding eigenfunction is given by $y(t)=t^{2}(1-t)$.

Example 4.2. Consider the boundary value problem

$$
y^{(4)}=\lambda t(2 y+1-t y-t), t \in(0,1) ; \quad y(0)=y^{\prime}(0)=y(1)=y^{\prime}(1)=0 .
$$

Here, $n=4, p=2$, and we let $H(t, y)=t^{2}(y+1)$ and $K(t, y)=t(2 y+1)$. With $f(y)=$ $y+1, h(t)=h_{1}(t)=t^{2}, k(t)=t, k_{1}(t)=2 t$, all the hypotheses (A1)-(A4) are satisfied. Also, $f_{0}=\infty$ and $f_{\infty}=1$. We have $\phi(s)=s^{2}(1-s)^{2}$ and $\int_{0}^{1} \phi(s) v(s) d s=\frac{1}{42}$. Hence it follows from Theorem 4.2 that $(0,42) \subseteq E$.

\section{Two positive solutions}

Throughout this section, we let $\lambda=1$ in the differential equation in (E).

Theorem 5.1. Let $w>0$ be given. Suppose that $f$ satisfies

$$
0<f(x) \leq w\left[\int_{0}^{1} \phi(s) v(s) d s\right]^{-1}, 0<x \leq w .
$$

(a) If $f_{0}=\infty$, then (E) has a positive solution $y_{1}$ with $0<\left\|y_{1}\right\| \leq w$;

(b) if $f_{\infty}=\infty$, then (E) has a positive solution $y_{2}$ with $\left\|y_{2}\right\| \geq w$;

(c) if $f_{0}=f_{\infty}=\infty$, then (E) has two positive solutions $y_{1}$ and $y_{2}$ with

$$
0<\left\|y_{3}\right\| \leq w \leq\left\|y_{2}\right\| .
$$

Proof. Of course, (c) follows from (a) and (b). To prove (a), we let

$$
A=\left\{\gamma \int_{\frac{1}{4}}^{\frac{3}{4}}(-1)^{n-p} G\left(\frac{1}{2}, s\right) u(s) d s\right\}^{-1} .
$$

Since $f_{0}=\infty$, there exists $0<r<w$ such that

$$
f(x) \geq A x, 0<x \leq r .
$$

Let $y \in C_{1}$ be such that $\|y\|=r$. On using (3.1) and (5.2) successively, we get

$$
S y\left(\frac{1}{2}\right) \geq A \int_{\frac{1}{4}}^{\frac{3}{4}}(-1)^{n-p} G\left(\frac{1}{2}, s\right) u(s) y(s) d s \geq\|y\| .
$$


This immediately implies that

$$
\|S y\| \geq\|y\| .
$$

If we set $\Omega_{1}=\{y \in B \mid\|y\|<r\}$, then (5.3) holds for $y \in C_{1} \cap \partial \Omega_{1}$. Next, let $y \in C_{\frac{1}{b}}$ be such that $\|y\|=w$. Then, in view of (3.1), Lemma 2.2 and (5.1) we find

$$
S y(t) \leq \int_{0}^{1} \phi(s) v(s) f(y(s)) d s \leq w=\|y\|, t \in[0,1] .
$$

Hence,

$$
\|S y\| \leq\|y\| .
$$

If we set $\Omega_{2}=\{y \in B \mid\|y\|<w\}$, then (5.4) holds for $y \in C_{2} \cap \partial \Omega_{2}$. Having obtained (5.3) and (5.4), it follows from Theorem 2.1 that $S$ has a fixed point $y_{1} \in C_{1} \cap\left(\bar{\Omega}_{2} \backslash \Omega_{1}\right)$ such that $r \leq\left\|y_{1}\right\| \leq w$. Clearly, this $y_{1}$ is a positive solution of (E).

Finally, we shall prove (c). As before, the condition (5.1) gives rise to (5.4). Hence, if we set $\Omega_{1}=\{y \in B \mid\|y\|<w\}$, then (5.4) holds for $y \in C_{\downarrow} \cap \partial \Omega_{1}$. Next, let

$$
M=\left\{\gamma \int_{\frac{1}{4}}^{\frac{3}{4}}(-1)^{n-p} G\left(\frac{1}{2}, s\right) u(s) d s\right\}^{-1} .
$$

Since $f_{\infty}=\infty$, we may choose $T>w$ such that

$$
f(x) \geq M x, x \geq T .
$$

Let $y \in C_{f}$ satisfy $\|y\|=\frac{T}{\gamma}$. Then $y(t) \geq \gamma\|y\|=\gamma \cdot \frac{T}{y}=T$ for $t \in\left[\frac{1}{4}, \frac{3}{4}\right]$, which in view of (5.5) leads to

$$
f(y(t)) \geq M y(t), t \in\left[\frac{1}{4}, \frac{3}{4}\right] .
$$

Using (3.1) and (5.6), we find

$$
S y\left(\frac{1}{2}\right) \geq M \int_{\frac{1}{4}}^{\frac{3}{3}}(-1)^{n-p} G\left(\frac{1}{2}, s\right) u(s) y(s) d s \geq\|y\| .
$$

Therefore, (5.3) holds. If we set $\Omega_{2}=\left\{y \in B \mid\|y\|<\frac{T}{7}\right\}$, then (5.3) holds for $y \in C_{1} \cap \partial \Omega_{2}$. Now that we have obtained (5.4) and (5.3), it follows from Theorem 2.1 that $S$ has a fixed point $y_{2} \in C_{1} \cap\left(\bar{\Omega}_{2} \backslash \Omega_{1}\right)$ such that $w \leq\left\|y_{2}\right\| \leq \frac{T}{y}$. It is clear that this $y_{2}$ is a positive solution of (E). 
Example 5.1. Let $M>0$ and consider the boundary value problem

$$
y^{(3)}=\frac{6}{t^{2}(1-t)^{4}+M}\left(y^{2}+M\right), t \in(0,1) ; \quad y(0)=y(1)=y^{\prime}(1)=0 .
$$

Here, $n=3, p=1$. Taking $f(y)=y^{2}+M$, we may choose $k(t)=k_{t}(t)=\frac{6}{r^{2}(1-t)^{4}+M}$ and $h(t)=h_{1}(t)=0$. It is obvious that $f_{0}=f_{\infty}=\infty$. We aim to find some $w>0$ such that condition (5.1) is fulfilled. For this, it is noted that

$$
\int_{0}^{1} \phi(s) v(s) d s=\int_{0}^{1} \frac{12 s(1-s)}{s^{2}(1-s)^{4}+M} d s \leq \int_{0}^{1} \frac{12 s(1-s)}{M} d s=\frac{2}{M}
$$

which implies

$$
\left[\int_{0}^{1} \phi(s) v(s) d s\right]^{-1} \geq \frac{M}{2}
$$

Since $f(x) \leq w^{2}+M$ for $0<x \leq w$, in view of (5.7) the condition (5.1) is fulfilled provided

$$
f(x) \leq w^{2}+M \leq w \frac{M}{2} \leq w\left[\int_{0}^{1} \phi(s) v(s) d s\right]^{-1}, 0<x \leq w
$$

which reduces to $2 w^{2}-w M+2 M \leq 0$. This inequality holds for some $w>0$ if and only if $M \geq 16$.

As an example, take $M=16$. Then, in order that (5.1) is satisfied, we set

$$
f(x) \leq w^{2}+16 \leq w\left[\int_{0}^{1} \phi(s) v(s) d s\right]^{-1}=8.01 w, 0<x \leq w .
$$

This leads to

$$
3.85 \leq w \leq 4.15 \text {. }
$$

Hence, (5.1) holds for any $w \in[3.85,4.15]$. By Theorem 5.1(c), there exist two positive solutions $y_{1}$ and $y_{2}$ with $0<\left\|y_{1}\right\| \leq w \leq\left\|y_{2}\right\|$. In view of (5.8), it is clear that $0<\left\|y_{1}\right\| \leq 3.85$ and $\left\|y_{2}\right\| \geq 4.15$. In fact, one positive solution is given by $y(t)=t(1-t)^{2}$, and we note that $\|y\|=y\left(\frac{1}{3}\right)=0.148$ is within the range given above.

\section{Two positive solutions of $\left(E_{1}\right)$}

Theorem 6.1. Let $w>0$ be given. Suppose that 


$$
\int_{0}^{1} s(1-s) a(s) d s \leq \frac{w}{w^{\alpha}+w^{\beta}} .
$$

Then, $\left(\mathrm{E}_{1}\right)$ has two positive solutions $y_{1}$ and $y_{2}$ such that

$$
0<\left\|y_{1}\right\| \leq w \leq\left\|y_{2}\right\| \text {. }
$$

Proof. Let $f(x)=x^{\alpha}+x^{\beta}$. Then, $f_{0}=f_{\infty}=\infty$. Further, we may take $k(t)=k_{1}(t)=$ $a(t)$ and $h(t)=h_{1}(t)=0$. Clearly, $f(x) \leq w^{\alpha}+w^{\beta}$ for $0<x \leq w$. So, to ensure that (5.1) is satisfied, we impose

$$
w^{\alpha}+w^{\beta} \leq w\left[\int_{0}^{1} \phi(s) v(s) d s\right]^{-1} .
$$

Here, $n=2, p=1$, so that $\phi(s)=s(1-s)$. Therefore, the above inequality is exactly (6.1). The conclusion follows from Theorem 5.1(c).

Remark 6.1. In [42] we have also discussed $\left(\mathrm{E}_{1}\right)$. The condition corresponding to (6.1) is obtained as [42] $\int_{0}^{1}(1-s) a(s) d s \leq \frac{w}{w^{3}+w^{\beta}}$. This is a stronger condition than (6.1), and hence (6.1) is an improvement.

Example 6.1. Consider $\left(\mathrm{E}_{1}\right)$, and let $w=1$. Then, (6.1) reduces to

$$
\int_{0}^{1} s(1-s) a(s) d s \leq \frac{1}{2}
$$

By Theorem 6.1, for those $a(t)$ which fulfill (6.2), $\left(\mathrm{E}_{1}\right)$ has double positive solutions $y_{1}$ and $y_{2}$ such that $0<\left\|y_{1}\right\| \leq 1 \leq\left\|y_{2}\right\|$. Some examples of such $a(t)$ are $3, t+2$, $\sin ^{2}(t+1)$.

Now, we shall establish upper and lower bounds for the two positive solutions of $\left(E_{1}\right)$.

Theorem 6.2. We define

$$
Q(x)=\frac{1}{2} \sup _{\delta \in\left(0, \frac{1}{2}\right)} \delta^{1+x}(1-2 \delta) a^{*}(\delta)
$$

where

$$
a^{*}(\delta)=\inf _{t \in[\delta, 1-\delta]} a(t)
$$


Let

$$
w_{1}=[Q(\alpha)]^{\frac{1}{-x}} \text { and } w_{2}=[Q(\beta)]^{\frac{1}{-\beta}}
$$

Let $w>0$ be given. Suppose that (6.1) holds. Then, $\left(\mathrm{E}_{1}\right)$ has twin positive solutions $y_{1}$ and $y_{2}$ such that

(a) if $w<\min \left\{w_{1}, w_{2}\right\}$, then $0<\left\|y_{1}\right\| \leq w \leq\left\|y_{2}\right\| \leq \min \left\{w_{1}, w_{2}\right\}$;

(b) if $\min \left\{w_{1}, w_{2}\right\}<w<\max \left\{w_{1}, w_{2}\right\}$, then

$$
\min \left\{w_{1}, w_{2}\right\} \leq\left\|y_{1}\right\| \leq w \leq\left\|y_{2}\right\| \leq \max \left\{w_{1}, w_{2}\right\}
$$

(c) if $w>\max \left\{w_{1}, w_{2}\right\}$, then $\max \left\{w_{1}, w_{2}\right\} \leq\left\|y_{1}\right\| \leq w \leq\left\|y_{2}\right\|$.

Proof. Since (6.1) is satisfied, it follows from Theorem 6.1 that $\left(E_{1}\right)$ has double positive solutions $y_{3}$ and $y_{4}$ such that

$$
0<\left\|y_{3}\right\| \leq w \leq\left\|y_{4}\right\|
$$

To establish upper and lower bounds for the two positive solutions, for an arbitrary $\delta \in\left(0, \frac{1}{2}\right)$, we let $C$ be a cone in $B$ defined by

$$
C=\left\{y \in B \mid y(t) \text { is nonnegative on }[0,1] ; \quad \min _{t \in[\delta, 1-\delta]} y(t) \geq \delta\|y\|\right\} .
$$

Define the operator $S: C \rightarrow B$ by

$$
S y(t)=\int_{0}^{1}-G_{1}(t, s) a(s)\left[y(s)^{\alpha}+y(s)^{\beta}\right] d s, t \in[0,1]
$$

where $G_{1}(t, s)=\left.G(t, s)\right|_{n=2, p=1}$. To obtain a positive solution of $\left(\mathrm{E}_{1}\right)$, we shall seek a fixed point of $S$ in the cone $C$. With the aid of Lemma 2.1 (note that there $\theta=\delta$ in our present case), it is easy to show that $S$ maps $C$ into itself. Also, the standard arguments yield that $S$ is completely continuous.

Let $y \in C$ be such that $\|y\|=w$. Then, in view of Lemma 2.2 (here $\phi(s)=s(1-s)$ ) and (6.1), we find for $t \in[0,1]$

$$
S y(t) \leq \int_{0}^{1} s(1-s) a(s)\left(w^{\alpha}+w^{\beta}\right) d s \leq w=\|y\| .
$$

Therefore, if we set $\Omega=\{y \in B \mid\|y\|<w\}$, then (5.4) holds for $y \in C \cap \partial \Omega$.

Now, let $y \in C$. It follows that 


$$
\begin{aligned}
\|S y\| & \geq \int_{0}^{1}-G_{1}(\delta, s) a(s)\left[y(s)^{\alpha}+y(s)^{\beta}\right] d s \\
& \geq \int_{\delta}^{1-\delta}-G_{1}(\delta, s) a^{*}(\delta)\left[y(s)^{\alpha}+y(s)^{\beta}\right] d s \\
& \geq \int_{\delta}^{1-\delta}-G_{1}(\delta, s) a^{*}(\delta)\left[\delta^{\alpha}\|y\|^{\alpha}+\delta^{\beta}\|y\|^{\beta}\right] d s .
\end{aligned}
$$

From (2.1), we have

$$
-G_{1}(\delta, s)=(1-s) \delta, s \in[\delta, 1]
$$

which we substitute into the above inequality, simplify and then take supremum over $\delta$ to obtain

$$
\|S y\| \geq Q(\alpha)\|y\|^{\alpha}+Q(\beta)\|y\|^{\beta} .
$$

Let $y \in C$ be such that $\|y\|=w_{1}$. Then, (6.7) provides

$$
\|S y\| \geq Q(\alpha)\|y\|^{\alpha}=Q(\alpha)\|y\|^{\alpha-1}\|y\|=\|y\| .
$$

If we set $\Omega_{1}=\left\{y \in B \mid\|y\|<w_{1}\right\}$, then (6.8) holds for $y \in C \cap \partial \Omega_{1}$. Now that we have obtained (5.4) and (6.8), it follows from Theorem 2.1 that $S$ has a fixed point $y_{5}$ such that

$$
\min \left\{w_{1}, w\right\} \leq\left\|y_{5}\right\| \leq \max \left\{w_{1}, w\right\} .
$$

Likewise, if we let $y \in C$ be such that $\|y\|=w_{2}$, then from (6.7) we get

$$
\|S y\| \geq Q(\beta)\|y\|^{\beta}=Q(\beta)\|y\|^{\beta-1}\|y\|=\|y\| .
$$

By setting $\Omega_{2}=\left\{y \in B \mid\|y\|<w_{2}\right\}$, we see that (6.10) holds for $y \in C \cap \partial \Omega_{2}$. Having obtained (5.4) and (6.10), by Theorem 2.1 we conclude that $S$ has a fixed point $y_{6}$ such that

$$
\min \left\{w_{2}, w\right\} \leq\left\|y_{6}\right\| \leq \max \left\{w_{2}, w\right\} .
$$

Now, a combination of (6.4), (6.9) and (6.11) yields our result. To be more precise, in Case (a) we may pick

$$
y_{1}=y_{3} \quad \text { and } \quad y_{2}= \begin{cases}y_{5}, & w_{1} \leq w_{2} \\ y_{6}, & w_{1} \geq w_{2}\end{cases}
$$

In Case (b), it is clear that

$$
y_{1}=\left\{\begin{array}{ll}
y_{5}, & w_{1} \leq w_{2} \\
y_{6}, & w_{1} \geq w_{2}
\end{array} \text { and } y_{2}= \begin{cases}y_{6}, & w_{1} \leq w_{2} \\
y_{5}, & w_{1} \geq w_{2}\end{cases}\right.
$$


Finally, in Case (c) we shall take

$$
y_{1}=\left\{\begin{array}{ll}
y_{6}, & w_{1} \leq w_{2} \\
y_{5}, & w_{1} \geq w_{2}
\end{array} \text { and } \quad y_{2}=y_{4}\right.
$$

Remark 6.2. In [42] by a different approach we also obtain similar upper and lower bounds for twin positive solutions of $\left(E_{1}\right)$. However, it has been noted in Remark 6.1 that the condition (6.1) in the present paper is an improvement.

Example 6.2. Consider the boundary value problem

$$
y^{\prime \prime}+\frac{2}{\left(t-t^{2}\right)^{0.2}+\left(t-t^{2}\right)^{1.2}}\left(y^{0.2}+y^{1.2}\right)=0, t \in(0,1) ; \quad y(0)=y(1)=0 .
$$

Here, $\alpha=0.2, \beta=1.2$ and $a(t)=\frac{2}{\left(t-t^{2}\right)^{0.2}+\left(t-t^{2}\right)^{1.2}}$. Condition (6.1) is equivalent to

$$
\frac{w}{w^{0.2}+w^{1.2}} \geq \int_{0}^{1} \frac{2 s(1-s)}{\left(s-s^{2}\right)^{0.2}+\left(s-s^{2}\right)^{1.2}} d s=0.391
$$

which is satisfied for any $w \in[0.525,103]$. Further, we find that $a^{*}(\delta)=a\left(\frac{1}{2}\right)$ and subsequently

$$
Q(x)=\frac{1}{2} \cdot a\left(\frac{1}{2}\right) \sup _{\delta \in\left(0, \frac{1}{2}\right)} \delta^{1+x}(1-2 \delta)=\frac{1}{2} \cdot a\left(\frac{1}{2}\right) \cdot\left[\frac{1+x}{2(2+x)}\right]^{1+x} \frac{1}{2+x}
$$

Thus,

$$
w_{1}=[Q(0.2)]^{\frac{1}{0.8}}=0.0569 \text { and } w_{2}=[Q(1.2)]^{-\frac{1}{0.2}}=3.23 \times 10^{7}
$$

Since $w \in\left(w_{1}, w_{2}\right)$, by Theorem 6.2(b) the problem has two positive solutions $y_{1}$ and $y_{2}$ such that $0.0569 \leq\left\|y_{1}\right\| \leq w \leq\left\|y_{2}\right\| \leq 3.23 \times 10^{7}$. Noting the range of $w$, this inequality leads to

$$
0.0569 \leq\left\|y_{1}\right\| \leq 0.525 \text { and } 103 \leq\left\|y_{2}\right\| \leq 3.23 \times 10^{7} .
$$

Indeed, a positive solution is given by $y(t)=t(1-t)$ and we note that $\|y\|=y\left(\frac{1}{2}\right)=0.25$ is within the range obtained in (6.12).

\section{Two positive solutions of $\left(E_{2}\right)$}

Theorem 7.1. Let $w>0$ be given. Suppose that

$$
\int_{0}^{1} s(1-s) a(s) d s \leq w e^{-\sigma w}
$$


Then, $\left(\mathrm{E}_{2}\right)$ has two positive solutions $y_{1}$ and $y_{2}$ such that

$$
0<\left\|y_{1}\right\| \leq w \leq\left\|y_{2}\right\| \text {. }
$$

Proof. Let $f(x)=e^{\sigma x}$. Then, $f_{0}=f_{\infty}=\infty$. Further, we may take $k(t)=k_{1}(t)=a(t)$ and $h(t)=h_{1}(t)=0$. It is clear that $f(x) \leq e^{\sigma w}$ holds for $0<x \leq w$. Therefore, (5.1) is satisfied provided that

$$
e^{\sigma w} \leq w\left[\int_{0}^{1} \phi(s) v(s) d s\right]^{-1}=w\left[\int_{0}^{1} s(1-s) a(s) d s\right]^{-1},
$$

i.e., condition (7.1) holds. The conclusion is immediate from Theorem 5.1(c).

Remark 7.1. In [42] we have also discussed $\left(E_{2}\right)$. The condition corresponding to (7.1) is obtained as [42] $\int_{0}^{1}(1-s) a(s) d s \leq w e^{-\sigma w}$. Clearly, this is a stronger condition than (7.1). Hence, (7.1) is an improvement.

Example 7.1. Consider the boundary value problem

$$
y^{\prime \prime}+a(t) e^{2 y}=0, t \in(0,1) ; \quad y(0)=y(1)=0 .
$$

Let $w=\frac{1}{2}$ be given. Then, condition (7.1) reduces to

$$
\int_{0}^{1} s(1-s) a(s) d s \leq \frac{1}{2 e}
$$

By Theorem 7.1, for those $a(t)$ which fulfill (7.2), $\left(E_{2}\right)$ has two positive solutions $y_{1}$ and $y_{2}$ such that $0<\left\|y_{1}\right\| \leq \frac{1}{2} \leq\left\|y_{2}\right\|$. Some examples of such $a(t)$ are $1, \cos ^{2}(t+1)$, $\frac{1}{2}(t+1)$.

Once again, we shall establish upper and lower bounds for the two positive solutions of $\left(E_{2}\right)$.

Theorem 7.2. Let $i \neq j$ be given integers in the set $\{0,2,3, \cdots\}$. We define

$$
R(x)=\frac{\sigma^{x}}{2(x !)} \sup _{\delta \in(0,1)} \delta^{1+x}(1-2 \delta) a^{*}(\delta)
$$

where $a^{*}(\delta)$ is given in (6.3), and let

$$
w_{1}=[R(j)]^{\text {尚 }} \text { and } w_{2}=[R(i)]^{\frac{1}{1-}} \text {. }
$$


Let $w>0$ be given. Suppose that (7.1) holds. Then. $\left(\mathrm{E}_{2}\right)$ has twin positive solutions $y_{1}$ and $y_{2}$ such that conclusions (a)-(c) of Theorem 6.2 hold.

Proof. Since (7.1) is fulfilled, it follows from Theorem 7.1 that $\left(\mathrm{E}_{2}\right)$ has double positive solutions $y_{3}$ and $y_{4}$ such that (6.4) holds.

To establish further upper and lower bounds for the two positive solutions, let $\delta \in\left(0, \frac{1}{2}\right)$ and $C$ be a cone in $B$ defined by (6.5). Further, we define the operator $S: C \rightarrow B$ by

$$
S y(t)=\int_{0}^{1}-G_{1}(t, s) a(s) e^{\sigma y(s)} d s, t \in[0,1]
$$

where $G_{1}(t, s)=\left.G(t, s)\right|_{n=2, p=1}$. To obtain a positive solution of $\left(\mathrm{E}_{2}\right)$, we shall seek a fixed point of $S$ in the cone $C$. As in the proof of Theorem 6.2, it can be verified that $S(C) \subseteq C$ and $S$ is completely continuous.

Let $y \in C$ be such that $\|y\|=w$. Using Lemma 2.2 (here $\phi(s)=s(1-s)$ ) and (7.1), we get for $t \in[0,1]$

$$
S y(t) \leq \int_{0}^{1} s(1-s) a(s) e^{\sigma y(s)} d s \leq \int_{0}^{1} s(1-s) a(s) e^{\sigma w} d s \leq w=\|y\| .
$$

Hence, if we set $\Omega=\{y \in B \mid\|y\|<w\}$, then (5.4) holds for $y \in C \cap \partial \Omega$.

Next, let $y \in C$. We find that

$$
\begin{aligned}
\|S y\| & \geq \int_{0}^{1}-G_{1}(\delta, s) a(s) e^{\sigma y(s)} d s \\
& \geq \int_{\delta}^{1-\delta}-G_{1}(\delta, s) a^{*}(\delta) e^{\sigma \gamma(s)} d s \geq \int_{\delta}^{1-\delta}-G_{1}(\delta, s) a^{*}(\delta) e^{\sigma \delta\|y\|} d s \\
& \geq \int_{\delta}^{1-\delta}-G_{1}(\delta, s) a^{*}(\delta)\left[\frac{(\sigma \delta)^{j}}{j !}\|y\|^{j}+\frac{(\sigma \delta)^{i}}{i !}\|y\|^{i}\right] d s
\end{aligned}
$$

where in the last inequality we have used the relation $e^{x} \geq \frac{x^{\prime}}{j !}+\frac{x^{i}}{i !}$ for $x>0$. On substituting the expression (6.6), we simplify and then take supremum over $\delta$ to get

$$
\|S y\| \geq R(j)\|y\|^{j}+R(i)\|y\|^{i} .
$$

Following a similar technique as in the proof of Theorem 6.2, from (7.3) we obtain (5.3) for $y \in C \cap \partial \Omega_{1}$ as well as for $y \in C \cap \partial \Omega_{2}$, where

$$
\Omega_{1}=\left\{y \in B \mid\|y\|<w_{1}\right\} \text { and } \Omega_{2}=\left\{y \in B \mid\|y\|<w_{2}\right\} \text {. }
$$

Now that we have obtained (5.3) and (5.4), by Theorem $2.1 S$ has a fixed point $y_{5}$ 
satisfying

$$
\min \left\{w_{1}, w\right\} \leq\left\|y_{5}\right\| \leq \max \left\{w_{1}, w\right\},
$$

and also a fixed point $y_{6}$ such that

$$
\min \left\{w_{2}, w\right\} \leq\left\|y_{6}\right\| \leq \max \left\{w_{2}, w\right\} .
$$

As in the proof of Theorem 6.2, a combination of (6.4), (7.4) and (7.5) yields conclusions (a)-(c) immediately.

Remark 7.2. By a different approach, similar upper and lower bounds are also obtained in [42] for twin positive solutions of $\left(\mathrm{E}_{2}\right)$. However, we have noted in Remark 7.1 that the condition (7.1) in the present paper is an improvement.

Example 7.2. Consider the boundary value problem

$$
y^{\prime \prime}+a e^{a y}=0, t \in(0,1) ; \quad y(0)=y(1)=0
$$

where $a, \sigma>0$. This problem has been well studied [1] and its solutions are

$$
y_{i}(t)=-\frac{2}{\sigma}\left\{\log \left[\cosh \left(\frac{c_{i}}{2}\left(t-\frac{1}{2}\right)\right)\right]-\log \left(\cosh \frac{c_{i}}{4}\right)\right\}
$$

where $c_{i}$ are solutions of the equation $c=\sqrt{2 a \sigma} \cosh \frac{c}{4}$.

Case 1: $a=1, \sigma=\frac{1}{2}, j=0, i=9$. It can be checked that condition (7.1) is satisfied provided that

$$
0.183 \leq w \leq 7.65
$$

Further, we find that $w_{1}=0.0625$ and $w_{2}=42.6$. Since $w \in\left(w_{1}, w_{2}\right)$, it follows from Theorem 7.2(b) that the boundary value problem has two positive solutions $y_{1}$ and $y_{2}$ with $0.0625 \leq\left\|y_{1}\right\| \leq w \leq\left\|y_{2}\right\| \leq 42.6$. In view of (7.7), we further have

$$
0.0625 \leq\left\|y_{1}\right\| \leq 0.183 \text { and } 7.65 \leq\left\|y_{2}\right\| \leq 42.6 \text {. }
$$

In fact, it is computed directly from (7.6) that $\left\|y_{1}\right\|=0.132$ and $\left\|y_{2}\right\|=10.3$.

In [42] the inequalities corresponding to (7.8) are found to be $0.0625 \leq\left\|y_{1}\right\| \leq 0.715$ and $4.31 \leq\left\|y_{2}\right\| \leq 42.6$. Clearly, (7.8) gives sharper bounds. This is due to the improvement of condition (7.1). if

Case 2: $a=7 \times 10^{-4}, \sigma=3, j=0, i=16$. By computation, condition (7.1) is fulfilled

$$
1.17 \times 10^{-4} \leq w \leq 3.42 .
$$




\section{RAVI P. AGARWAL, MARTIN BOHNER AND PATRICIA J. Y. WONG}

Further, we find that $w_{1}=4.38 \times 10^{-5}$ and $w_{2}=11.5$. Since $w \in\left(w_{1}, w_{2}\right)$, by Theorem 7.2(b) the boundary value problem has double positive solutions $y_{1}$ and $y_{2}$ with $4.38 \times 10^{-5} \leq\left\|y_{1}\right\| \leq w \leq\left\|y_{2}\right\| \leq 11.5$. Once again in view of (7.9), it follows that

$$
4.38 \times 10^{-5} \leq\left\|y_{1}\right\| \leq 1.17 \times 10^{-4} \text { and } 3.42 \leq\left\|y_{2}\right\| \leq 11.5 \text {. }
$$

In fact, it is computed from (7.6) that $\left\|y_{1}\right\|=8.75 \times 10^{-5}$ and $\left\|y_{2}\right\|=4.02$.

Corresponding to (7.10), in [42] we obtain $4.38 \times 10^{-5} \leq\left\|y_{1}\right\| \leq 3.50 \times 10^{-4}$ and $3.02 \leq\left\|y_{2}\right\| \leq 11.5$ which are not as sharp as (7.10). Again, this illustrates the improvement of condition (7.1).

\section{REFERENCES}

1. R. P. Agarwal, Boundary Value Problems for Higher Order Differential Equations (World Scientific, Singapore, 1986).

2. R. P. Agarwal and P. J. Y. Wong, Existence of solutions for singular boundary value problems for higher order differential equations. Rend. Sem. Mat. Fis. Milano 65 (1995), 249-264.

3. R. P. Agarwal and P. J. Y. Wong, Advanced Topics in Difference Equations (Kluwer Academic Publishers, Dordrecht, 1997).

4. D. Aronson, M. G. Crandall and L. A. Peletier, Stabilization of solutions of a degenerate nonlinear diffusion problem, Nonlinear Anal. 6 (1982), 1001-1022.

5. F. Atici and A. C. Peterson, Bounds for positive solutions for a focal boundary value problem, in Advances in Difference Equations II, Comput. Math. Appl, to appear.

6. C. Bandle, C. V. Coffman and M. Marcus, Nonlinear elliptic problems in annular domains, J. Differential Equations 69 (1987), 322-345.

7. C. Bandle and M. K. Kwong, Semilinear elliptic problems in annular domains, J. Appl. Math. Phys. 40 (1989), 245-257.

8. Y. S. ChOI and G. S. LUDFord, An unexpected stability result of the near-extinction diffusion flame for non-unity lewis numbers, Quart. J. Mech. Appl. Math. 42 (1989), 143-158.

9. C. J. Chyan and J. Henderson, Positive solutions for singular higher order nonlinear equations, Diff. Eqs. Dyn. Sys. 2 (1994), 153-160.

10. D. S. COHEN, Multiple stable solutions of nonlinear boundary value problems arising in chemical reactor theory, SIAM J. Appl. Math. 20 (1971), 1-13.

11. E. N. DANCER, On the structure of solutions of an equation in catalysis theory when a parameter is large, J. Differential Equations 37 (1980), 404-437.

12. P. W. Eloe and J. Henderson, Positive solutions for $(n-1,1)$ boundary value problems, Nonlinear Anal. 28 (1997), 1669-1680.

13. P. W. Eloe and J. Henderson, Positive solutions and nonlinear $(k, n-k)$ conjugate eigenvalue problems.

14. L. H. ERBE, S. Hu and H. WANG, Multiple positive solutions of some boundary value problems, J. Math. Anal. Appl. 184 (1994), 640-648.

15. L. H. ERBE and H. WANG, On the existence of positive solutions of ordinary differential equations, Proc. Amer. Math. Soc. 120 (1994), 743-748. 
16. A. M. Fink, The radial Laplacian Gel'fand problem, in Delay and Differential Equations (Ames, IA, 1991, World Sci. Publishing, River Edge, NJ, 1992), 93-98.

17. A. M. Fink, J. A. Gatica and G. E. Hernandez, Eigenvalues of generalized Gel'fand models, Nonlinear Anal. 20 (1993), 1453-1468.

18. H. Fujita, On the nonlinear equations $\delta u+e^{u}=0$ and $\frac{\partial v}{\alpha x}=\delta v+e^{0}$, Bull. Amer. Math. Soc. 75 (1969), 132-135.

19. X. GARAIZAR, Existence of positive radial solutions for semilinear elliptic problems in the annulus, $J$. Differential Equations 70 (1987), 69-72.

20. J. A. Gatica, V. Oliker and P. Waltman, Singular nonlinear boundary value problems for second-order ordinary differential equations, J. Differential Equations 79 (1989), 62-78.

21. I. M. Gel'Fand, Some problems in the theory of quasilinear equations, Uspehi Mat. Nauka 14 (1959), 87-158. English translation, Trans. Amer. Math. Soc. 29 (1963), 295-381.

22. D. Guo and V. Lakshmikantham, Nonlinear Problems in Abstract Cones (Academic Press, San Diego, 1988).

23. G. B. GuSTAFSON, A Green's function convergence principle, with applications to computation and norm estimates, Rocky Mountain J. Math. 6 (1976), 457-492.

24. D. Hankerson and A. C. Peterson, Comparison of eigenvalues for focal point problems for $n$-th order difference equations, Differential Integral Equations 3 (1990), 363-380.

25. J. Henderson, Singular boundary value problems for difference equations, Dynamic Systems Appl. 1 (1992), 271-282.

26. J. HeNDERSON, Singular boundary value problems for higher order difference equations, V. Lakshmikantham, ed., in Proceedings of the First World Congress on Nonlinear Analysts (Walter de Gruyter and Co., 1996), 1139-1150.

27. M. A. Krasnosel'skiI, Positive Solutions of Operator Equations (Noordhoff, Groningen, 1964).

28. F. Merdivenci, Two positive solutions of a boundary value problem for difference equations. J. Differ. Equations Appl. 1 (1995), 262-270.

29. F. Merdivenci, Green's matrices and positive solutions of a discrete boundary value problem, Panamer. Math. J. 5 (1995), 25-42.

30. F. Merdivenci, Positive solutions for focal point problems for $2 n$-th order difference equations, Panamer. Math. J. 5 (1995), 71-82.

31. D. O'Regan, Theory of Singular Boundary Value Problems (World Scientific, Singapore, 1994).

32. S. PARTER, Solutions of differential equations arising in chemical reactor processes, $S I A M$ J. Appl. Math. 26 (1974), 687-716.

33. A. C. PETERson, Boundary value problems for an $n$-th order difference equation, SIAM J. Math. Anal. 15 (1984), 124-132.

34. $\mathrm{H}$. WANG, On the existence of positive solutions for semilinear elliptic equations in the annulus, J. Differential Equations 109 (1994), 1-7.

35. P. J. Y. Wong and R. P. Agarwal, Eigenvalues of boundary value problems for higher order differential equations, Mathematical Problems in Engineering 2 (1996), 401-434. 


\section{RAVI P. AGARWAL, MARTIN BOHNER AND PATRICIA J. Y. WONG}

36. P. J. Y. Wong and R. P. Agarwal, Double positive solutions of $(n, p)$ boundary value problems for higher order difference equations, Comput. Math. Appl. 32 (8) (1996), 1-21.

37. P. J. Y. Wong and R. P. Agarwal, On the existence of solutions of singular boundary value problems for higher order difference equations, Nonlinear Anal. 28 (1997), 277-287.

38. P. J. Y. Wong and R. P. Agarwal, On the existence of positive solutions of higher order difference equations, Topological Methods in Nonlinear Analysis 10 (1997), 339-351.

39. P. J. Y. Wong and R. P. Agarwal, On the eigenvalues of boundary value problems for higher order difference equations, Rocky Mountain J. Math., to appear.

40. P. J. Y. Wong and R. P. Agarwal, Eigenvalue characterization for $(n, p)$ boundary value problems, J. Austral. Math. Soc. Ser. B. 39 (1998), 386-407.

41. P. J. Y. Wong and R. P. Agarwal, Eigenvalues of an $n$th order difference equation with $(n, p)$ type conditions, Dynam. Contin. Discrete Impuls. Systems 4 (1998), 149-172.

42. P. J. Y. Wong and R. P. Agarwal, On eigenvalues and twin positive solutions of $(n, p)$ boundary value problems, Functional Differential Equations 4 (1997), 443-476.

43. P. J. Y. Wong and R. P. Agarwal, Extension of continuous and discrete inequalities due to Eloe and Henderson, Nonlinear Anal. 34 (1998), 479-487.

AgARWAL AND BoHNER

NATIONAL UNIVERSITY OF SINGAPORE

Department OF Mathematics

10 Kent Ridge Crescent

SINGAPORE 119260

E-mail address: matravip@leonis.nus.sg martin@saturn.sdsu.edu
WONG

NANYANG TEChNOLOGICAL UNIVERSITY

Division of Mathematics

469 BuKit TIMAH RoAD

SINGA PORE 259756

E-mail address: wongjyp@nievax.nie.ac.sg 